\title{
Treatment satisfaction with paliperidone extended-release tablets: open-label study in schizophrenia patients dissatisfied with previous antipsychotic medication
}

\author{
This article was published in the following Dove Press journal: \\ Neuropsychiatric Disease and Treatment \\ 13 April 2017 \\ Number of times this article has been viewed
}

\author{
Fu De Yang' \\ Juan $\mathrm{Li}^{\prime}$ \\ Yun Long Tan' \\ Wei Ye Liang' \\ Rongzhen Zhang' \\ Ning Wang' \\ Wei Feng' \\ Shangli $\mathrm{Cai}^{2}$ \\ Jian Min Zhuo ${ }^{2}$ \\ Li Li Zhang ${ }^{2}$ \\ 'Beijing Hui-Long-Guan Hospital, \\ ${ }^{2}$ Department of Medical Affairs, Xian \\ Janssen Pharmaceutical Ltd, Beijing, \\ People's Republic of China
}

Objective: The aim of this study was to evaluate the changes in treatment satisfaction after switching to paliperidone extended-release (ER) in Chinese schizophrenia patients dissatisfied with their previous antipsychotic treatment.

Methods: In this 8-week, open-label, single-arm, multicenter, prospective study, 1,693 patients dissatisfied with previous antipsychotic medication were enrolled and switched to paliperidone ER tablets (3-12 mg/d) based on clinical judgment. The primary efficacy end point was change in Medication Satisfaction Questionnaire (MSQ) score from baseline to week 8 . The secondary end points included percentage of patients with MSQ score $\geq 4$, as well as changes in Clinical Global Improvement-Severity (CGI-S) and Personal and Social Performance (PSP) scores.

Results: MSQ scores increased significantly from baseline (mean [standard deviation $\{\mathrm{SD}\}$ ]: 2.48 [0.55]) to week 8 (5.47 [0.89], $P<0.0001$; primary end point, full analysis set). The percentage of patients with MSQ score $\geq 4$ was $95.9 \%$ at week 8 , indicating that most of the patients were satisfied with their treatment. Significant $(P<0.0001)$ improvements from baseline to week 8 were noted in CGI-S score (2.37 [1.20]) and PSP score (25.5 [15.0]). A total of 174 (10.28\%) patients experienced adverse events (AEs). The most common ( $>10$ patients) events were extrapyramidal disorder $(n=84,4.96 \%)$, poor quality sleep $(n=18,1.06 \%)$ and akathisia $(n=13$, $0.77 \%$ ). The majority of AEs were mild to moderate in severity. No deaths occurred.

Conclusion: Treatment satisfaction improved after switching to paliperidone ER from the previous antipsychotic in Chinese patients with schizophrenia.

Keywords: atypical antipsychotics, open label, paliperidone extended-release, schizophrenia, medical satisfaction questionnaire, patient satisfaction, Chinese

\section{Introduction}

There has been an increasing awareness in the past few years over the role of patient subjective satisfaction with antipsychotic treatment in the overall success of the therapeutic intervention. ${ }^{1,2}$ Patient satisfaction with the treatment is now considered a clinical index of efficacy, treatment adherence and quality of life in schizophrenia. ${ }^{3-6}$ Even with the advent of numerous treatment options, discontent with treatment can result in relapse and aggravation of the symptoms of schizophrenia, leading to worsening of illness. ${ }^{6,7}$ The major influencing factors of patient satisfaction with an antipsychotic treatment have been identified to be efficacy, treatment side effects, patient's psychological state and lack of involvement in treatment planning. ${ }^{8-10}$ 
Discontinuation and frequent switching of antipsychotic medication in patients with schizophrenia occur due to lack of efficacy, tolerability, partial or nonadherence to medication, relapse despite adherence to medication, impaired functioning or patients' willingness to change the current treatment. ${ }^{11-13}$ Switching antipsychotic treatment due to unsuccessful treatment with an antipsychotic can be an intricate decision. Moreover, the challenges include deterioration or relapse of psychosis; exacerbation of autonomic, behavioral and extrapyramidal symptoms (EPSs); worsening of other adverse effects; and impairment of medication adherence as the result of complex switching procedures. ${ }^{14}$

Paliperidone extended-release (paliperidone ER), marketed as Invega ${ }^{\circledR}$ (Janssen Pharmaceutica, Beerse, Belgium), is a once-daily atypical antipsychotic tablet formulation approved for the treatment of schizophrenia in the People's Republic of China in 2009. ${ }^{15}$ Paliperidone is the major active metabolite of risperidone, and it acts as an antagonist of dopamine-2 (D2) receptors and 5-hydroxytryptamine 2A (5-HT2A) receptors, with a higher affinity for 5-HT2A receptors. ${ }^{15,16} \mathrm{Via}$ an osmotic-controlled-release oral delivery system, paliperidone ER delivers at a controlled rate over 24 hours, leading to a gradual rise in plasma concentration after the initial dose, reducing plasma drug fluctuations at steady state, with once-daily dosing. ${ }^{16,17}$ In addition, paliperidone ER undergoes limited hepatic metabolism (59\% of the compound is excreted unchanged in urine), no substantial metabolism by the cytochrome P450 2D6 (CYP2D6) pathway and has no active metabolites. ${ }^{16}$ These features of paliperidone ER may benefit patients and improve their satisfaction with antipsychotic treatment.

This study aimed to evaluate treatment satisfaction, efficacy, tolerability and social functioning in patients with schizophrenia who were dissatisfied with previous antipsychotics and switched to paliperidone ER based on physicians' judgment.

\section{Methods}

\section{Study participants}

Key inclusion criteria included the following: men or women, aged 18-65 years, who met the criteria for schizophrenia diagnosis based on the International Statistical Classification of Diseases and Related Health Problems (ICD)-10, Diagnostic and Statistical Manual of Mental Disorders-IV or the Chinese Classification of Mental Disorders-3, with Medication Satisfaction Questionnaire (MSQ) score of $\leq 3$, dissatisfaction with previous treatment and advice from their physician to switch to paliperidone ER. Patients had received either a single antipsychotic at the minimum effective dose for at least 4 consecutive weeks before enrollment, or at least 2 antipsychotic drugs, with 1 of the antipsychotic drugs administered at the minimum effective dose for at least 4 consecutive weeks and the other $\operatorname{drug}(\mathrm{s})$ administered at less than minimum effective dose within a week before enrollment. The following doses were used as references: risperidone $2 \mathrm{mg}$; olanzapine $5 \mathrm{mg}$; quetiapine $150 \mathrm{mg}$; ziprasidone $40 \mathrm{mg}$; aripiprazole $10 \mathrm{mg}$; chlorpromazine by oral administration $200 \mathrm{mg}$; chlorpromazine by intramuscular injection $25 \mathrm{mg}$; haloperidol by oral administration $40 \mathrm{mg}$; haloperidol by intramuscular injection $5 \mathrm{mg}$; amisulpride $400 \mathrm{mg}$; perphenazine $8 \mathrm{mg}$. The reference doses of other unlisted drugs were determined by investigators according to the package insert of the drug.

Exclusion criteria included previous treatment with clozapine, long-acting injection antipsychotics and paliperidone ER within a month before enrollment into the study; use of drugs that cause prolonged QTc interval, as recorded in an electrocardiogram (ECG), such as class IA and class III antiarrhythmic drugs (quinidine, amiodarone, quinolone antibiotics such as gatifloxacin, chlorpromazine and thioridazine); known hypersensitivity to risperidone or paliperidone; and pregnancy or breastfeeding status of women.

The ethics committee of Beijing Hui Long Guan Hospital approved the protocol, and the study was conducted in accordance with the ethical principles that have their origin in the Declaration of Helsinki and that are consistent with Good Clinical Practices and applicable regulatory requirements. All patients provided written informed consent before participation.

\section{Study design and treatment}

This was a nonrandomized, open-label, single-arm, phase-4, multicenter, prospective, 8-week study conducted across 38 centers in the People's Republic of China from June 2011 to September 2012. The study included the screening/baseline phase (day -1$)$ and a treatment period of 8 weeks.

Eligible patients were treated with paliperidone ER dose ranging from $3 \mathrm{mg} / \mathrm{d}$ to $12 \mathrm{mg} / \mathrm{d}$ based on physician's discretion after considering patients' clinical history and symptoms. Patients treated with risperidone could be directly switched to paliperidone ER; patients treated with highly potent conventional antipsychotics (such as haloperidol), ziprasidone and amisulpride needed at least 1-week titration period; and patients treated with low-potency conventional antipsychotics (such as chlorpromazine), olanzapine and quetiapine needed 
at least 2 weeks' titration period. Patients previously treated with adjunctive antipsychotics were required to switch from primary antipsychotic to paliperidone ER within 1 month; whether to use a secondary adjunctive antipsychotic was decided by clinical judgment.

\section{Clinical outcomes}

The primary efficacy end point was change from baseline to week 8 in the MSQ score (7-point scale: 1= extremely dissatisfied, $2=$ very dissatisfied, $3=$ somewhat dissatisfied, $4=$ neither satisfied nor dissatisfied, $5=$ somewhat satisfied, $6=$ very satisfied and $7=$ extremely satisfied) to measure paliperidone ER treatment satisfaction in patients with schizophrenia.

Secondary efficacy end points included percentage of patients with MSQ score $\geq 4$ at week 8 , evaluation of symptom improvement as measured by decrease in Clinical Global Impression-Severity (CGI-S) score, percentage of patients with CGI-S score $\leq 3$ at week 8 , improvement of functioning - as measured by increase in Personal and Social Performance (PSP) score from baseline to week 8 and percentage of patients with PSP score $\geq 71$ at week 8 .

\section{Subgroup analyses}

Subgroup analyses to evaluate efficacy, tolerability and personal and social functioning as measured by MSQ, CGI-S and PSP scores were performed after subgrouping patients based on reasons for switching (dissatisfaction with social functioning, dissatisfaction with efficacy and dissatisfaction with safety), antipsychotic drug use at baseline (olanzapine, risperidone, quetiapine, aripiprazole, ziprasidone, chlorpromazine and others) and severities of the disease. Patients were classified into 3 categories according to the CGI-S score at baseline: mild subgroup (borderline mentally ill + mildly ill), moderate subgroup (moderately ill + markedly ill) and severe subgroup (severely ill + extremely ill). Correlation analyses between MSQ and CGI-S scores, as well as between MSQ and PSP scores, were also conducted.

\section{Sample size}

According to previous data, ${ }^{2}$ estimated MSQ score improvement was expected to be $2.4 \pm 1.4$; a sample size of 87 patients provided a precision of 0.3 of half-width for $95 \%$ confidence interval. Since the objective of this study was to explore the MSQ score improvement with this defined precision level in every identified subgroup of patients with prior exposure to different antipsychotics (including olanzapine, risperidone, quetiapine, aripiprazole and ziprasidone), the number of patients in the smallest subgroup was expected to be no less than 87 . Based on the estimation that the smallest fraction of patients with prior antipsychotic treatment was about $4 \%$ for aripiprazole and ziprasidone treatment groups, a total sample size of $2,175(87 / 4 \%=2,175)$ was needed to meet the sample size requirements mentioned. Considering a dropout rate of $20 \%$, a total of 2,867 patients were to be recruited.

\section{Statistical analysis}

All statistical tests were 2 -tailed, and $P$-values $\leq 0.05$ were considered statistically significant. A paired $t$-test or Wilcoxon signed-rank test was used as a difference test for the change in the baseline and postbaseline values depending on whether the data followed normal distribution.

\section{Analysis sets}

Efficacy analyses were based on full analysis set (FAS), which included all patients who received at least 1 dose of paliperidone ER and had at least 1 postbaseline efficacy evaluation. All patients who received at least 1 dose of paliperidone ER and had at least 1 safety evaluation were included into the safety set (SS).

\section{Results}

\section{Patient disposition}

In this study, 1,694 patients were enrolled, of whom 1,186 patients comprised the FAS (Figure 1). Nineteen patients withdrew from the study due to loss to follow-up $(n=6,0.35 \%)$, poor efficacy $(n=5,0.29 \%)$, safety and tolerability issue $(n=3,0.18 \%)$, withdrawal of consent $(n=2,0.12 \%)$ and other $(n=3,0.18 \%)$. Demographic and baseline characteristics were generally balanced, with 586 men (49.41\%) and 600 women $(50.59 \%)$ (Table 1$)$. The mean (standard deviation [SD]) age was 34.93 (13.40) years, mean (SD) schizophrenia history was 4.98 (7.61) years. The mean (SD) frequency of recurrence was 2.65 (2.62). As most subgroups of patients (except the ziprasidone group) with prior antipsychotic exposure enrolled more than 87 patients, the study was terminated early.

A total of $1,061(89.46 \%)$ patients were using a single antipsychotic before entering the study. The most commonly used single antipsychotics were olanzapine (26.98\%), risperidone $(21.67 \%)$, quetiapine (14.84\%), aripiprazole (11.47\%) and ziprasidone (4.3\%). Approximately 10.39\% $(n=123)$ patients were on concomitant antipsychotics. The most commonly used primary drug was olanzapine $(2.95 \%)$, risperidone (2.36\%) and aripiprazole (1.52\%). Psychoactive drugs were used by 134 patients $(11.30 \%)$, mainly including 


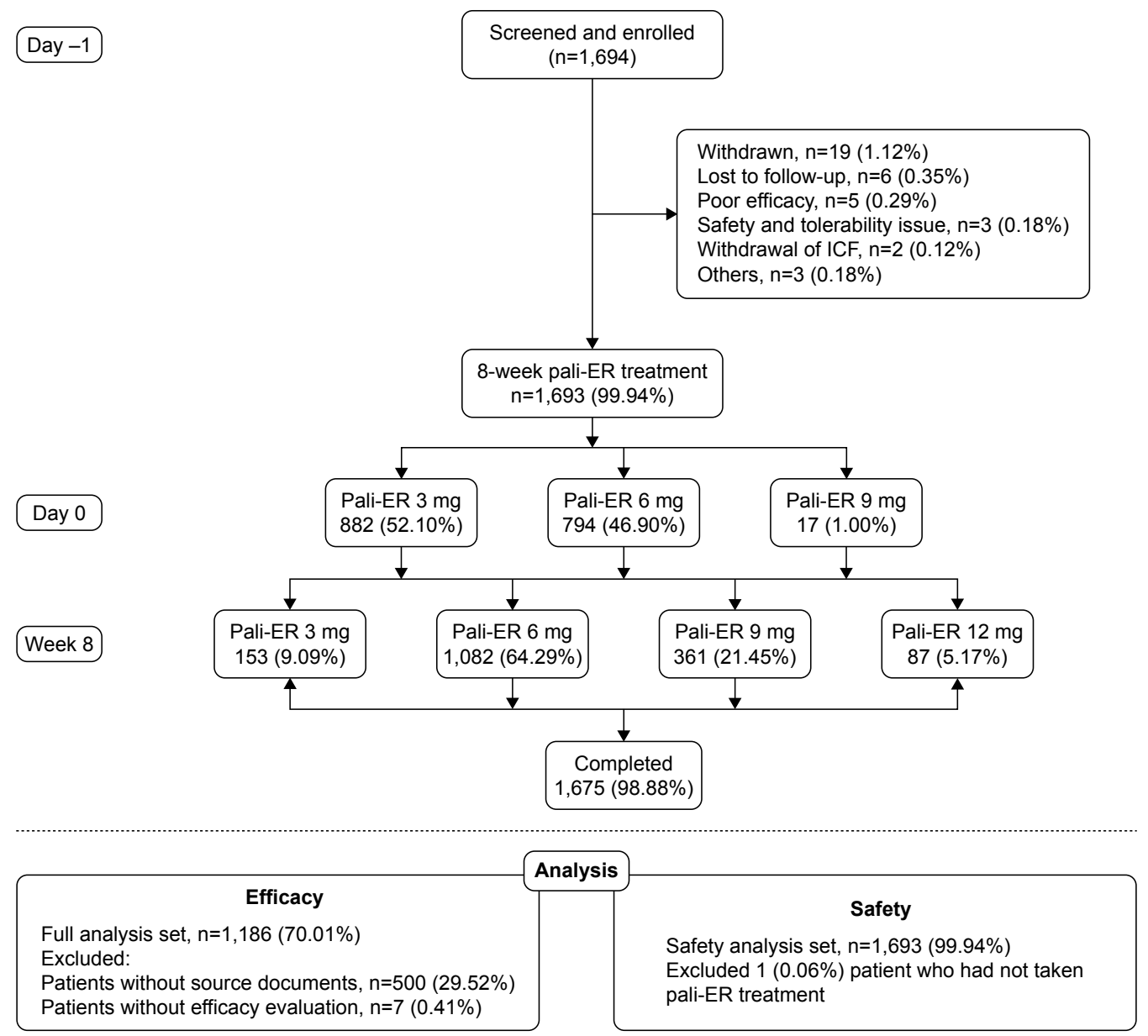

Figure I Summary of patient disposition and treatment.

Abbreviations: ICF, informed consent form; pali-ER, paliperidone extended-release tablets.

Table I Demographics and baseline characteristics

\begin{tabular}{|c|c|}
\hline Parameters & Overall $(\mathrm{N}=\mathrm{I}, 186)$ \\
\hline Age, years, mean (SD) & $34.93(13.40)$ \\
\hline \multicolumn{2}{|l|}{ Sex, n (\%) } \\
\hline Men & $586(49.4 I)$ \\
\hline Women & $600(50.59)$ \\
\hline \multicolumn{2}{|c|}{ History of schizophrenia, years } \\
\hline $\mathrm{N}$ & 1,186 \\
\hline Mean (SD) & $4.98(7.61)$ \\
\hline $95 \% \mathrm{Cl}$ & $4.55-5.42$ \\
\hline \multicolumn{2}{|l|}{ Frequency of recurrence } \\
\hline $\mathrm{n}$ (missing) & $\mathrm{I}, 013(173)$ \\
\hline Mean (SD) & $2.65(2.62)$ \\
\hline $95 \% \mathrm{Cl}$ & $2.49-2.81$ \\
\hline \multicolumn{2}{|c|}{ Types of previous antipsychotics with adequate dosage and duration } \\
\hline $\mathrm{n}$ (missing) & $1,163(23)$ \\
\hline Mean (SD) & $1.96(1.21)$ \\
\hline $95 \% \mathrm{Cl}$ & $1.89-2.03$ \\
\hline \multicolumn{2}{|c|}{ Schizophrenia types, n (\%) } \\
\hline Paranoid type & $742(62.56)$ \\
\hline Hebephrenic type & $53(4.47)$ \\
\hline \multicolumn{2}{|l|}{ (disorganized type) } \\
\hline Catatonic type & $21(1.77)$ \\
\hline Simple type & $16(1.35)$ \\
\hline Unknown & $354(29.85)$ \\
\hline
\end{tabular}

Abbreviations: $\mathrm{Cl}$, confidence interval; SD, standard deviation. sedative hypnotics $(4.05 \%)$, mood stabilizers $(3.63 \%)$, antidepressants (2.78\%) and anxiolytics (1.69\%). Previous adverse reactions were treated in 180 patients, mainly by anticholinergic (13.83\%), $\beta$-receptor blocker $(1.43 \%)$ and antihistamine $(0.08 \%)$ drugs.

\section{Concomitant treatment}

Of the 1,693 patients included in the SS, concomitant drugs including all drugs except paliperidone ER were used by $445(26.28 \%)$ patients, most of which acted on the central nervous system ( $\mathrm{n}=424,25.04 \%)$ followed by cardiovascular and hematopoietic system $(\mathrm{n}=37,2.19 \%)$, digestive system $(\mathrm{n}=13,0.77 \%)$ and the endocrine and metabolic system $(n=11,0.65 \%)$.

\section{Treatment exposure}

Of the 1,693 patients, $882(52.10 \%), 794(46.90 \%)$ and $17(1.00 \%)$ patients initiated paliperidone ER with 3, 6 and $9 \mathrm{mg} / \mathrm{d}$, respectively, and 153 (9.09\%) patients took paliperidone ER at the dose of $3 \mathrm{mg} / \mathrm{d}, 1,082$ (64.29\%) took $6 \mathrm{mg} / \mathrm{d}$, 
$361(21.45 \%)$ took $9 \mathrm{mg} / \mathrm{d}$ and $87(5.17 \%)$ took $12 \mathrm{mg} / \mathrm{d}$ at week 8 (Figure 1).

\section{Primary efficacy}

At week 8, mean (SD) MSQ score increased significantly $(P<0.0001)$ from baseline (mean [SD] increase: 2.99 [1.05]) (Table 2).

\section{Secondary efficacy}

At baseline, 1,155 (97.39\%) patients had "very dissatisfied" and "somewhat dissatisfied" ratings, and 23 (1.94\%) patients were extremely dissatisfied with previous treatment (Table 3). After switching to paliperidone ER for 8 weeks, the majority of patients (1,018 [85.83\%]) expressed "somewhat satisfied" and "very satisfied" rating. None of the patients expressed "extreme dissatisfaction" with paliperidone ER treatment.

The proportion of patients with MSQ score $\geq 4$ was 95.87\% at week 8 (Table 3). Paliperidone ER treatment significantly reduced disease severity $(P<0.0001)$, as demonstrated by reduction in the CGI-S score. Compared with baseline, the mean (SD) CGI-S scores at week 8 decreased significantly by $2.37(1.20)(P<0.0001$; Table 4$)$. The number of patients reporting normalcy increased to $17 \%$ at week 8 compared to $<1 \%$ in baseline. After 8 weeks' treatment with paliperidone ER, the percentage of patients with CGI-S score $\leq 3$ was $83.22 \%$. These data indicate that the number of patients who had schizophrenia remission gradually increased during 8-week paliperidone ER treatment, and the degrees of symptoms returned to normal or mild.

Personal and social functioning significantly improved at week 8 compared with that at baseline (mean [SD] change: 25.5 [15.03], $P<0.0001$ ) (Table 5). More than 50\% of the patients had clinically significant improvement after receiving 8-week paliperidone ER, with $55.48 \%$ patients reporting PSP score of $\geq 71$.

\section{Subgroup analysis}

Based on switching reasons at baseline, approximately $37 \%$ of patients expressed dissatisfaction with social functioning, $34 \%$ of patients expressed dissatisfaction with efficacy and $28 \%$ of patients expressed dissatisfaction with safety. At week 8, the change in mean (SD) MSQ scores from baseline was 3.03 (1.04) in subgroup dissatisfied with safety, 3.04 (1.05) in subgroup dissatisfied with efficacy and 3.00 (1.05) in subgroup dissatisfied with social functioning $(P<0.0001$; Table 2$)$. Percentage of patients with MSQ score $\geq 4$ increased to $97 \%$ after 8 weeks' treatment with paliperidone ER at each subgroup based on switching reasons, showing a significant treatment satisfaction with paliperidone ER. Similar improvement in personal and social performance and decrease in disease severity was also observed in this subgroup at week 8 (Tables 4 and 5).

Based on the use of previous antipsychotics such as risperidone, quetiapine, aripiprazole, ziprasidone and chlorpromazine, the increase of mean (SD) MSQ scores from baseline to week 8 were 2.94 (1.09) in the olanzapine subgroup, 2.92 (1.04) in the risperidone subgroup, 2.94 (1.05) in the quetiapine subgroup, 3.04 (1.02) in the aripiprazole subgroup, 3.19 (1.17) in the ziprasidone subgroup, 3.12 (0.88) in the chlorpromazine subgroup and 3.21 (1.03) in the other drug subgroup. There were statistically significant differences between baseline and week 8 among each subgroup ( $P<0.0001$; Table 2$)$. In the subgroup based on antipsychotics used at baseline, the percentage of patients who were satisfied with treatment increased from $<2 \%$ to $>90 \%$ from baseline to week 8 after switching to paliperidone ER.

Table 2 MSQ scores at baseline and week 8 (full analysis set)

\begin{tabular}{|c|c|c|c|c|c|}
\hline \multirow[t]{2}{*}{ Parameters } & \multirow[t]{2}{*}{ n (\%) } & \multicolumn{3}{|c|}{ MSQ score, mean (SD) } & \multirow[t]{2}{*}{$P$-value } \\
\hline & & Baseline & Week 8 & Week 8 - baseline & \\
\hline Full analysis set, $\mathrm{N}=\mathrm{I}, \mathrm{I} 86$ & & $2.48(0.55)$ & $5.47(0.89)$ & $2.99(1.05)$ & $<0.0001$ \\
\hline \multicolumn{6}{|c|}{ Subgrouped by different reasons for switching drug } \\
\hline Dissatisfied with safety & $739(27.98)$ & $2.48(0.56)$ & $5.51(0.87)$ & $3.03(1.04)$ & $<0.0001$ \\
\hline Dissatisfied with efficacy & $906(34.31)$ & $2.42(0.55)$ & $5.47(0.88)$ & $3.04(1.05)$ & $<0.0001$ \\
\hline Dissatisfied with social functioning & $987(37.37)$ & $2.47(0.55)$ & $5.47(0.88)$ & $3.00(1.05)$ & $<0.0001$ \\
\hline \multicolumn{6}{|c|}{ Subgrouped by antipsychotics used at baseline } \\
\hline Olanzapine & $355(29.98)$ & $2.5 \mathrm{I}(0.57)$ & $5.44(0.92)$ & $2.94(1.09)$ & $<0.000$ I \\
\hline Risperidone & $285(24.07)$ & $2.57(0.54)$ & $5.49(0.92)$ & $2.92(1.04)$ & $<0.0001$ \\
\hline Quetiapine & 192 (16.22) & $2.45(0.52)$ & $5.40(0.82)$ & $2.94(1.05)$ & $<0.0001$ \\
\hline Aripiprazole & I54 (I3.0I) & $2.4 I(0.5 I)$ & $5.45(0.91)$ & 3.04 (1.02) & $<0.0001$ \\
\hline Ziprasidone & $54(4.56)$ & $2.30(0.54)$ & $5.48(0.93)$ & $3.19(1.17)$ & $<0.0001$ \\
\hline Chlorpromazine & $43(3.63)$ & $2.44(0.59)$ & $5.56(0.70)$ & $3.12(0.88)$ & $<0.0001$ \\
\hline Other & 101 (8.53) & $2.39(0.57)$ & $5.59(0.85)$ & $3.21(1.03)$ & $<0.0001$ \\
\hline
\end{tabular}

Abbreviations: MSQ, Medication Satisfaction Questionnaire; SD, standard deviation. 
Table 3 Summary of MSQ assessment and percentages of patients with MSQ score $\geq 4$ at baseline and week 8 (full analysis set)

\begin{tabular}{|c|c|c|}
\hline Responses, $\mathrm{N}=\mathrm{I}, \mathrm{I} 86$ & n (\%) & $95 \% \mathrm{Cl}$ \\
\hline \multicolumn{3}{|l|}{ Baseline } \\
\hline Extremely dissatisfied & $23(1.94)$ & \\
\hline Very dissatisfied & $58 \mid(48.99)$ & \\
\hline Somewhat dissatisfied & $574(48.40)$ & \\
\hline Neither satisfied nor dissatisfied & $8(0.67)$ & \\
\hline \multicolumn{3}{|l|}{ Week 8} \\
\hline Very dissatisfied & $19(1.60)$ & \\
\hline Somewhat dissatisfied & $30(2.53)$ & \\
\hline Neither satisfied nor dissatisfied & $88(7.42)$ & \\
\hline Somewhat satisfied & $322(27.16)$ & \\
\hline Very satisfied & $696(58.68)$ & \\
\hline Extremely satisfied & $31(2.6 I)$ & \\
\hline \multicolumn{3}{|l|}{ Baseline } \\
\hline Patients with MSQ score $\geq 4$ & $8(0.67)$ & $0.21-1.14$ \\
\hline \multicolumn{3}{|l|}{ Week 8} \\
\hline Patients with MSQ score $\geq 4$ & I, I 37 (95.87) & $94.74-97.00$ \\
\hline
\end{tabular}

Abbreviations: $\mathrm{Cl}$, confidence interval; MSQ, Medication Satisfaction Questionnaire.

Similar improvement in personal and social performance and decrease in disease severity was also observed in this subgroup at week 8 (Tables 4 and 5).

Correlation analysis showed that MSQ and CGI-S scores were negatively correlated (correlation coefficient $=-0.55$, $P<0.0001$ ), whereas MSQ and PSP scores were positively correlated (correlation coefficient $=0.43, P<0.0001$ ).

\section{Safety}

A total of $174(10.28 \%)$ patients experienced adverse events (AEs), and most were of mild-to-moderate severity. Extrapyramidal disorder (4.96\%), poor quality sleep $(1.06 \%)$, akathisia $(0.77 \%)$ and insomnia $(9[0.53 \%])$ were most commonly reported AEs (Table 6). AEs related to hyperprolactinemia were reported in 5 patients. The majority (88.37\%) of AEs required no change in dosage. A total of 115 AEs were resolved, 84 had remission, 14 had no obvious change and 2 became aggravated at week 8 . Two patients discontinued paliperidone ER treatment due to AEs. Serious $\mathrm{AE}$ of muscle tone disorder was observed in 1 woman, and another patient experienced tardive dyskinesia. No deaths occurred during the study.

\section{Discussion}

In this study, there was a significant improvement in treatment satisfaction in patients treated with paliperidone ER. Mean (SD) MSQ score improved 2.99 (1.05) points, and up to $95 \%$ of patients expressed treatment satisfaction, with MSQ score $\geq 4$. Similar results were reported by Canuso et al, ${ }^{2}$ with mean (SD) MSQ score improvement from 2.7 (0.8) (veryto-somewhat dissatisfied) at baseline to 5.1 (1.2) (somewhat satisfied) at end point $(P<0.001)$, and $82.7 \%$ patients were satisfied with their medication at end point.

Patient satisfaction with an antipsychotic treatment is influenced by multiple factors, including efficacy of the treatment, drug side effects, patient's functional status and psychosocial factors. Better efficacy of the treatment is strongly correlated with high level of patient satisfaction, and occurrence of side effects is strongly correlated with low level of satisfaction. ${ }^{10}$ Many studies have demonstrated that atypical antipsychotics are significantly associated with better patient satisfaction compared to typical antipsychotics. ${ }^{10,18,19}$

Paliperidone ER is an atypical antipsychotic that needs only once-daily administration, thus reducing plasma drug fluctuations at steady state. ${ }^{16,17}$ In addition, it undergoes

Table 4 CGI-S score at baseline and week 8 (full analysis set)

\begin{tabular}{|c|c|c|c|c|c|}
\hline \multirow[t]{2}{*}{ Parameters } & \multirow[t]{2}{*}{ n (\%) } & \multicolumn{3}{|c|}{ CGI-S score, mean (SD) } & \multirow[t]{2}{*}{$P$-value } \\
\hline & & Baseline & Week 8 & Week 8 - baseline & \\
\hline Full analysis set, $\mathrm{N}=\mathrm{I}, \mathrm{I} 86$ & & $4.95(0.95)$ & $2.58(1.10)$ & $-2.37(1.20)$ & $<0.000$ \\
\hline \multicolumn{6}{|c|}{ Subgrouped by different reasons for switching drug } \\
\hline Dissatisfied with safety & 739 (27.98) & $4.84(0.92)$ & $2.51(1.06)$ & $-2.33(1.18)$ & $<0.000$ \\
\hline Dissatisfied with efficacy & $906(34.31)$ & $5.11(0.88)$ & $2.61(1.10)$ & $-2.50(1.19)$ & $<0.000$ \\
\hline Dissatisfied with social functioning & $987(37.37)$ & $4.93(0.93)$ & $2.55(1.08)$ & $-2.38(1.18)$ & $<0.0001$ \\
\hline \multicolumn{6}{|c|}{ Subgrouped by antipsychotics used at baseline } \\
\hline Olanzapine & $355(29.98)$ & $4.83(1.00)$ & $2.57(1.08)$ & $-2.26(1.22)$ & $<0.0001$ \\
\hline Risperidone & $285(24.07)$ & $4.88(0.97)$ & $2.48(1.14)$ & $-2.40(1.27)$ & $<0.000$ \\
\hline Quetiapine & $192(16.22)$ & $5.04(0.88)$ & $2.76(1.02)$ & $-2.28(1.14)$ & $<0.000$ I \\
\hline Aripiprazole & 154 (I3.0I) & $5.12(0.82)$ & $2.43(0.98)$ & $-2.69(1.17)$ & $<0.000$ I \\
\hline Ziprasidone & $54(4.56)$ & $4.91(0.85)$ & $2.50(1.08)$ & $-2.4 I(0.90)$ & $<0.000$ \\
\hline Chlorpromazine & $43(3.63)$ & $5.23(0.97)$ & $2.70(1.12)$ & $-2.53(1.24)$ & $<0.000$ I \\
\hline Other & I0I (8.53) & $5.03(1.00)$ & $2.78(1.31)$ & $-2.25(1.21)$ & $<0.000 \mid$ \\
\hline
\end{tabular}

Abbreviations: CGI-S, Clinical Global Impression-Severity; SD, standard deviation. 
Table 5 PSP score at baseline and week 8 (full analysis set)

\begin{tabular}{|c|c|c|c|c|c|}
\hline \multirow[t]{2}{*}{ Parameters } & \multirow[t]{2}{*}{ n (\%) } & \multicolumn{3}{|c|}{ PSP score, mean (SD) } & \multirow[t]{2}{*}{$P$-value } \\
\hline & & Baseline & Week 8 & Week 8 - baseline & \\
\hline Full analysis set, $\mathrm{N}=\mathrm{I}, \mathrm{I} 86$ & & $46.2(14.94)$ & $71.7(12.01)$ & $25.5(I 5.30)$ & $<0.0001$ \\
\hline \multicolumn{6}{|c|}{ Subgrouped by different reasons for switching drug } \\
\hline Dissatisfied with safety & $739(27.98)$ & $47.34(14.61)$ & $72.68(11.98)$ & $25.34(14.73)$ & $<0.0001$ \\
\hline Dissatisfied with efficacy & $906(34.31)$ & $44.70(14.46)$ & $70.96(11.44)$ & $26.26(14.85)$ & $<0.0001$ \\
\hline Dissatisfied with social functioning & $987(37.37)$ & $46.39(14.84)$ & $71.95(11.66)$ & $25.56(14.85)$ & $<0.0001$ \\
\hline \multicolumn{6}{|c|}{ Subgrouped by antipsychotics used at baseline } \\
\hline Olanzapine & $355(29.98)$ & $47.37(14.85)$ & $71.35(11.13)$ & $23.99(14.85)$ & $<0.0001$ \\
\hline Risperidone & $285(24.07)$ & $47.04(15.16)$ & $72.66(13.27)$ & $25.62(15.75)$ & $<0.0001$ \\
\hline Quetiapine & $192(16.22)$ & $44.73(15.20)$ & $69.76(11.38)$ & $25.03(\mid 4.7 I)$ & $<0.0001$ \\
\hline Aripiprazole & 154 (13.0I) & 45.32 (I3.23) & $72.66(9.90)$ & 27.34 (I3.29) & $<0.0001$ \\
\hline Ziprasidone & $54(4.56)$ & $48.54(15.58)$ & 73.89 (14.94) & 25.35 (13.09) & $<0.000$ I \\
\hline Chlorpromazine & $43(3.63)$ & $44.56(16.78)$ & $70.58(12.11)$ & $26.02(16.09)$ & $<0.0001$ \\
\hline Other & I0I (8.53) & 43.11 (15.15) & $72.02(13.34)$ & 28.91 (16.78) & $<0.000$ I \\
\hline
\end{tabular}

Abbreviations: PSP, Personal and Social Performance; SD, standard deviation.

limited hepatic metabolism, thereby reducing the risks of hepatic drug-drug and drug-disease interactions. ${ }^{16} \mathrm{~A}$ recent network meta-analysis, comparing the efficacy, acceptability and tolerability of 8 atypical antipsychotics in Chinese patients with acute schizophrenia, demonstrated paliperidone ER to be in a position of superior efficacy with lower discontinuation risk. ${ }^{20}$ Another systematic review summarizing the clinical evidence of the efficacy, effectiveness and safety of paliperidone in the Chinese population reported that paliperidone ER demonstrated superior improvement in the PSP scores and at least comparable efficacy with active comparators assessed..$^{15}$ The documented efficacy and safety profile of paliperidone ER and its positive effect on functional status may contribute to the improvement in patient satisfaction with the treatment.

The MSQ is a single-item questionnaire that evaluates satisfaction with antipsychotic medication in schizophrenia

Table 6 Summary of adverse events (safety analysis set)

\begin{tabular}{ll}
\hline Adverse events, overall $\mathbf{N}=\mathbf{I , 6 9 3}$ & $\mathbf{n}(\%)$ \\
\hline Any adverse event & $\mathrm{I} 74(\mathbf{I 0 . 2 8})$ \\
Any serious adverse event & $\mathrm{I}(0.06)$ \\
Treatment stopped due to adverse event & $2(0.12)$ \\
Common adverse events that took place in $>5$ patients on & \\
paliperidone ER & \\
Extrapyramidal disorder & $84(4.96)$ \\
Poor quality sleep & $18(1.06)$ \\
Akathisia & $13(0.77)$ \\
Tardive dyskinesia & $5(0.30)$ \\
Blood prolactin increased & $5(0.30)$ \\
Insomnia & $9(0.53)$ \\
Amenorrhea & $5(0.30)$ \\
Tachycardia & $5(0.30)$ \\
\hline
\end{tabular}

Abbreviation: ER, extended release. patients. Use of patient-reported outcome to evaluate medication satisfaction is a well-accepted tool in nonpsychotic illnesses; in recent years, use of such tools to evaluate the effectiveness of schizophrenia treatment has gained popularity. Vernon et $\mathrm{al}^{21}$ and other researchers ${ }^{22,23}$ evaluated the psychometric properties of the MSQ in psychotic populations taking paliperidone ER vs placebo and found that the responses to this single-question item were able to separate patients receiving active drug from those receiving placebo and was therefore a good proxy to assess both efficacy and tolerability features of schizophrenia treatment from a patient perspective.

The secondary efficacy results from this study also demonstrated that after switching from previous antipsychotics to paliperidone ER, there was a significant improvement in the personal and social functioning of the patients (PSP score mean [SD] change: 25.5 [15.03]), with up to $56 \%$ patients achieving the PSP score $\geq 71$ at week 8 ; this observation was consistent with that in other studies. ${ }^{24,25}$ In a study conducted by Patrick et al ${ }^{26}$ using data from 3 randomized phase-3 studies, the effectiveness of paliperidone ER with respect to PSP score using multiple analysis methods, such as betweengroup minimum important difference estimates, responder analyses, between-group cumulative frequency comparisons of PSP score change from baseline to end point and numberneeded-to treat estimates, showed a meaningful improvement in PSP score across the dose range of $3-15 \mathrm{mg} / \mathrm{d}$. One of the essential components of optimizing good outcome of schizophrenia is improvement in social functioning. Even with good symptom control, poor personal and social functioning can compromise the potential of patients to optimize their ability 
to function in daily life. Therefore, the impact of antipsychotic treatment on functioning is an important factor for improving the prognosis of patients with schizophrenia. ${ }^{26}$

In our study, a notable decrease in disease severity was also observed after 8 weeks of paliperidone ER treatment compared to that at baseline. Nearly $83 \%$ patients had remission with respect to symptoms associated with schizophrenia, with disease severity changing from severely ill/moderately ill to mildly ill or normal. The number of patients showing improvement was greater than that observed in previous studies. ${ }^{27-29}$ A favorable response was also demonstrated in the subgroup analysis (subgroups: reasons for switching treatment, antipsychotic use and disease severities), showing that treatment with paliperidone ER improved treatment satisfaction, disease severity and social functioning of the Chinese patients in this study.

During the 8-week paliperidone ER treatment, the safety and tolerability profile observed was similar to that seen in earlier studies by Savitz et al, ${ }^{30} \mathrm{Si}$ et al, ${ }^{25}$ Nakagawa et al, ${ }^{24}$ and Singh et al, ${ }^{29}$ with EPSs, akathisia and insomnia as common AEs associated with paliperidone. Furthermore, the incidences of AEs in the current study were relatively lower than those observed in other similar studies; ${ }^{23,25,31,32}$ this may be attributed to the low dosage of paliperidone ER used in this study, ie, $\sim 64 \%$ patients were receiving $6 \mathrm{mg} / \mathrm{d}$ dose at week 8 . In this study, AEs were either reported by patients spontaneously or were obtained by means of interviewing them in a nondirected manner at study visits. Patients visited the study center only at 2 time points during the study (at baseline and at end point), which may have led to some reporting bias, because many AEs may have happened within the first 2 weeks after switching to another antipsychotic. This is one of the limitations of the study.

Other major limitations of this study include its openlabel design and lack of an active or placebo comparator. The shorter duration of the study is another limitation, especially because a longer follow-up period would have been more appropriate to explore the sustained effectiveness. In addition, lack of psychopathological assessments (such as Positive and Negative Syndrome Scale [PANSS] scores), as well as the lack of cognitive assessments, anthropometric and metabolic parameters, and prolactin data, were also considered as a limitation of the study.

\section{Conclusion}

Treatment switch to paliperidone ER in Chinese patients with schizophrenia resulted in significant patient subjective satisfaction, along with improvement in psychotic symptoms and social functioning, and no new safety concerns were identified.

\section{Acknowledgments}

The authors thank Ashwini Patil, MS (SIRO Clinpharm Pvt Ltd; certified medical publication professional of the International Society for Medical Publication Professionals [ISMPP $\mathrm{CMPP}^{\mathrm{TM}}$ ]) for drafting the manuscript; Himabindu Gutha, $\mathrm{PhD}$ (SIRO Clinpharm Pvt Ltd), for assisting in addressing journal responses and editing of manuscript; and Ellen Baum, PhD (Janssen Research \& Development, LLC) for editorial assistance in the preparation of this manuscript. The authors thank the study participants, without whom this study would not have been accomplished, as well as the investigators for their participation in this study. This study was funded by Xian Janssen Pharmaceutical Ltd.

\section{Author contributions}

Fu De Yang was the principal investigator for the study. Juan Li, Yun Long Tan, Wei Ye Liang, Rongzhen Zhang, Ning Wang and Wei Feng were involved in the study design and protocol development, conduct of the study and interpretation of the data. Shangli Cai and Li Li Zhang were involved in the data interpretation. Jian Min Zhuo was responsible for the statistical analyses. All authors contributed toward data analysis, drafting and revision of the paper and agree to be accountable for all aspects of the work.

\section{Disclosure}

Fu De Yang has been a consultant and has received grant support from Xian Janssen Pharmaceutical Ltd. Shangli Cai was an employee of Xian Janssen Pharmaceutical Ltd, China, at the time of study and can be currently contacted at 3D Medicines, Department of Medical Affairs, Building 2, Block B, 158 XinJunhuan Street, Pujiang Hi-tech Park, Shanghai 201114, China. Li Li Zhang is an employee of Xian Janssen Pharmaceutical Ltd, and Jian Min Zhuo is an employee of Johnson \& Johnson (China) Investment Ltd, both of which are part of the family of Johnson \& Johnson Company. The authors report no other conflicts of interest in this work.

\section{References}

1. McCabe R, Saidi M, Priebe S. Patient-reported outcomes in schizophrenia. Br J Psychiatry Suppl. 2007;50:s21-s28.

2. Canuso CM, Grinspan A, Kalali A, et al. Medication satisfaction in schizophrenia: a blinded-initiation study of paliperidone extended release in patients suboptimally responsive to risperidone. Int Clin Psychopharmacol. 2010;25(3):155-164.

3. Gharabawi GM, Lasser RA, Bossie CA, Zhu Y, Amador X. Insight and its relationship to clinical outcomes in patients with schizophrenia or schizoaffective disorder receiving long-acting risperidone. Int Clin Psychopharmacol. 2006;21(4):233-240.

4. Mohamed S, Rosenheck R, McEvoy J, Swartz M, Stroup S, Lieberman JA. Cross-sectional and longitudinal relationships between insight and attitudes toward medication and clinical outcomes in chronic schizophrenia. Schizophr Bull. 2009;35(2):336-346. 
5. Haro JM, Salvador-Carulla L. The SOHO (Schizophrenia Outpatient Health Outcome) study: implications for the treatment of schizophrenia. CNS Drugs. 2006;20(4):293-301.

6. Jones PB, Barnes TRE, Davies L, et al. Randomized controlled trial of the effect on quality of life of second- vs first-generation antipsychotic drugs in schizophrenia. Cost Utility of the Latest Antipsychotic Drugs in Schizophrenia Study (CULASS 1). Arch Gen Psychiatry. 2006;63(10): 1079-1087.

7. Lieberman J, Stroup T, McEvay J, et al. Effectiveness of antipsychotic drugs in patients with chronic schizophrenia. NEnglJMed.2005;353(12): 1209-1223.

8. Kalali A. Patient satisfaction with, and acceptability of, atypical antipsychotics. Curr Med Res Opin. 1999;15(2):135-137.

9. Rofail D, Gray R, Gournay K. The development and internal consistency of the Satisfaction with Antipsychotic Medication Scale. Psychol Med. 2005;35(7):1063-1072.

10. Chue P. The relationship between patient satisfaction and treatment outcomes in schizophrenia. J Psychopharmacol. 2006; 20(6 suppl):38-56.

11. Peuskens J, Rubio G, Schreiner A. Dosing and switching of paliperidone ER in patients with schizophrenia: recommendations for clinical practice. Ann Gen Psychiatry. 2014;13(1):10.

12. Higashi K, Medic G, Littlewood KJ, Diez T, Granström O, De Hert M. Medication adherence in schizophrenia: factors influencing adherence and consequences of nonadherence, a systematic literature review. Ther Adv Psychopharmacol. 2013;3(4):200-218.

13. Roussidis A, Kalkavoura C, Dimelis D, et al. Reasons and clinical outcomes of antipsychotic treatment switch in outpatients with schizophrenia in real-life clinical settings: the ETOS observational study Ann Gen Psychiatry. 2013;12(1):42.

14. NHS. Guidelines for Antipsychotic Medication Switches. 2009. Available from: http://www.humber.nhs.uk/Downloads/Services/Pharmacy/ Guidelines/Antipsychotic\%20medication \%20switches\%20guidelines. pdf. Accessed February 27, 2017.

15. Zhang L, Li J, Zhao Y, Su Y, Si T. Critical evaluation of paliperidone in the treatment of schizophrenia in Chinese patients: a systematic literature review. Neuropsychiatr Dis Treat. 2016;12:113-131.

16. Invega ${ }^{\circledR}$ Sustenna ${ }^{\circledR}$ extended-release injectable suspension [prescribing Information]. Titusville, NJ: Janssen Pharmaceuticals, Inc.; 2014.

17. Conley R, Gupta SK, Sathyan G. Clinical spectrum of the osmoticcontrolled release oral delivery system (OROS), an advanced oral delivery form. Curr Med Res Opin. 2006;22(10):1879-1892.

18. Gasquet I, Tcherny-Lessenot S, Lepine JP, Falissard B. Patient satisfaction with psychotropic drugs: sensitivity to change and relationship to clinical status, quality-of-life, compliance and effectiveness of treatment. Results from a nation-wide 6-month prospective study. Eur Psychiatry. 2006;21(8):531-538.

19. Nordon C, Rouillon F, Barry C, Gasquet I, Falissard B. Determinants of treatment satisfaction of schizophrenia patients: results from the ESPASS study. Schizophr Res. 2012;139:211-217.
20. Bai Z, Wang G, Cai S, et al. Efficacy, acceptability and tolerability of 8 atypical antipsychotics in Chinese patients with acute schizophrenia: a network meta-analysis. Schizophr Res. Epub 2017 Jan 17.

21. Vernon MK, Revicki DA, Awad AG, et al. Psychometric evaluation of the Medication Satisfaction Questionnaire (MSQ) to assess satisfaction with antipsychotic medication among schizophrenia patients. Schizophr Res. 2010;118(1-3):271-278

22. Gharabawi GM, Greenspan A, Rupnow M, et al. Reduction in psychotic symptoms as a predictor of patient satisfaction with antipsychotic medication in schizophrenia: data from a randomized double-blind trial. BMC Psychiatry. 2006;6:45.

23. Sun L, Zhou T, Lei T. Efficacy and safety of paliperidone ER in the treatment of adolescent patients with first-episode schizophrenia. Chinese J New Drugs. 2011;9:009.

24. Nakagawa R, Ohnishi T, Kobayashi H, et al. The social functional outcome of being naturalistically treated with paliperidone extendedrelease in patients with schizophrenia. Neuropsychiatr Dis Treat. 2015; 22:1511-1521.

25. Si T, Tan Q, Zhang K, Wang Y, Rui Q. An open-label, flexible-dose study of paliperidone extended-release in Chinese patients with firstonset psychosis. Neuropsychiatr Dis Treat. 2015;11:87-95.

26. Patrick DL, Burns T, Morosini P, Gagnon DD, Rothman M, Adriaenssen I. Measuring social functioning with the personal and social performance scale in patients with acute symptoms of Schizophrenia: interpretation of results of a pooled analysis of three Phase III trials of paliperidone extended-release tablets. Clin Ther. 2010;32(2):275-292.

27. Conde BL, Sionzon MP, Benedicto EG. A post-marketing surveillance study on the effectiveness and safety of paliperidone ER among Filipino adult patients diagnosed with schizophrenia. Acta Medica Phillipina. 2013;47(2).

28. Turkoz I, Bossie CA, Lindenmayer JP, Schooler N, Canuso CM. Paliperidone ER and oral risperidone in patients with schizophrenia: a comparative database analysis. BMC Psychiatry. 2011;11:21.

29. Singh J, Robb A, Ujjwala V, et al. A randomized double blind study of paliperidone extended release in treatment of acute schizophrenia in adolescent. Biol Phychiatry. 2011;70(12):1179-1187.

30. Savitz AJ, Lane R, Nuamah I, Gopal S, Hough D. Efficacy and safety of paliperidone extended release in adolescents with schizophrenia: a randomized, double-blind study. $J$ Am Acad Child Adolesc Psychiatry. 2015;54(2):126-137.

31. Zhang Y, Dai G. Efficacy and metabolic influence of paliperidone ER, aripiprazole and ziprasidone to patients with first-episode schizophrenia through 52 weeks follow-up in China. Hum Psychopharmacol.2012;27(6): 605-614.

32. Yu YM, Li HF, Yang PD. Efficacy, safety and tolerability of paliperidone ER treatment on acute schizophrenia at flexible dosage. Shanghai Jingshen Yixue. 2010;22:349-353.
Neuropsychiatric Disease and Treatment

\section{Publish your work in this journal}

Neuropsychiatric Disease and Treatment is an international, peerreviewed journal of clinical therapeutics and pharmacology focusing on concise rapid reporting of clinical or pre-clinical studies on a range of neuropsychiatric and neurological disorders. This journa is indexed on PubMed Central, the 'PsycINFO' database and CAS,

\section{Dovepress}

and is the official journal of The International Neuropsychiatric Association (INA). The manuscript management system is completely online and includes a very quick and fair peer-review system, which is all easy to use. Visit http://www.dovepress.com/testimonials.php to read real quotes from published authors. 